\title{
Research of the influence of propeller root on the aerodynamic characteristics of propeller
}

\author{
Yuan-Qiang LIU ${ }^{1,2, a}$, Song XIANG ${ }^{2, b}$ and Gang TONG ${ }^{2, c}$ \\ ${ }^{1}$ Shenyang Aircraft Design Institute, Shenyang 110035, China; \\ ${ }^{2}$ Liaoning Key Laboratory of General Aviation, Shenyang Aerospace University, Shenyang 110136 , \\ China. \\ aliuyuanqiang1988@163.com; Xs7432@sina.com; ${ }^{\mathrm{c} T o n g r e n @ s a u . e d u . c n ~}$
}

Keywords: Propeller, aerodynamic characteristics, propeller root, wind tunnel test.

\begin{abstract}
In this paper the wind tunnel test is used to analyze the effect of root chord of propeller on aerodynamic performance. There were two distributions of the chord near hub of propeller, all parameters are same except the maximum chord. Two models are respectively made of carbon fiber and beech. From the wind tunnel test results, the following conclusions can be drawn: By increasing the root chord within a reasonable range, thrust and torque will be increased, which also causes the efficiency to increase by $10 \%$. Although the carbon fiber material is very strong for making propeller, we also cannot ignore the influence of root chord.
\end{abstract}

\section{Introduction}

The propeller produces forward thrust for the aircraft. The optimization of the propulsion system becomes important. Through improvements of the configuration and geometry of the propeller, an increase of the propulsive efficiency and a decrease of the operating cost can be achieved. Due to the rapid development of carbon fiber and the increase of flight range , particularly in general aviation, the geometry shape of the appropriate propeller becomes different. The root chord of carbon fiber propeller is shorter than wooden propeller. With considering these factors, the significance to measure the influence is intensified substantially. The performance of the propeller will reduce around the root portion of the propeller. And the vortices formed on the propeller's hub reduce the efficiency. The strength of the vortices depends on the axial load distribution on the propeller and the geometry near the hub ${ }^{[1]}$.

Many researchers had studied the relation between propeller performances and geometry. A suitable design of minimum Induced Loss Propellers has already been put forward by the Larrabee ${ }^{[2]}$ in 1979.Eppler and Hepperle ${ }^{[3]}$ used inverse methods to design propeller. Angelo S D, Berardi F et al ${ }^{[4]}$ put forward aerodynamic performances of propellers with parametric considerations on the optimal design. XIANG Song, ZHANG Li-guo et al ${ }^{[5]}$ raised a design method for high efficiency propeller. LIU Yuan-qiang, GUO Jin-suo et al ${ }^{[6]}$ emphasized that the aerodynamic of propeller root chord cannot been ignored. A. B. HAINES et al used Tunnel Tests on a Rotor Wooden Spitfire Propeller and Data applied to Single Radius Calculations ${ }^{[7]}$. J. Cecrdle researched and analyzed the Influence of Propeller Blade Lift Distribution on Whirl Flutter Stability Characteristics ${ }^{[8]}$.

In this article, the performance of propeller root is evaluated by changing one parameter: the chord length. This article shows two propellers at a series of velocities and rotated with constant rotational speed of the wind tunnel.

\section{Experimental content and method}

Two 0.6:1 laboratory scale experimental models were set-up for the evaluation of aerodynamic parameters. Diameter is $0.96 \mathrm{~m}$, two blades. All parameters are same except the maximum chord. In order to evaluate the effect of a different size of root chord, propeller parameters were given in Tab.1. 1\# propeller was made by carbon fiber.2\# propeller was made by beech. 
Tab.1 parameter of propeller

\begin{tabular}{|c|c|c|}
\hline number & Pitch angle & Maximum chord \\
\hline $1 \#$ & $15^{\circ}$ & $150 \mathrm{~mm}$ \\
\hline $2 \#$ & $15^{\circ}$ & $170 \mathrm{~mm}$ \\
\hline
\end{tabular}

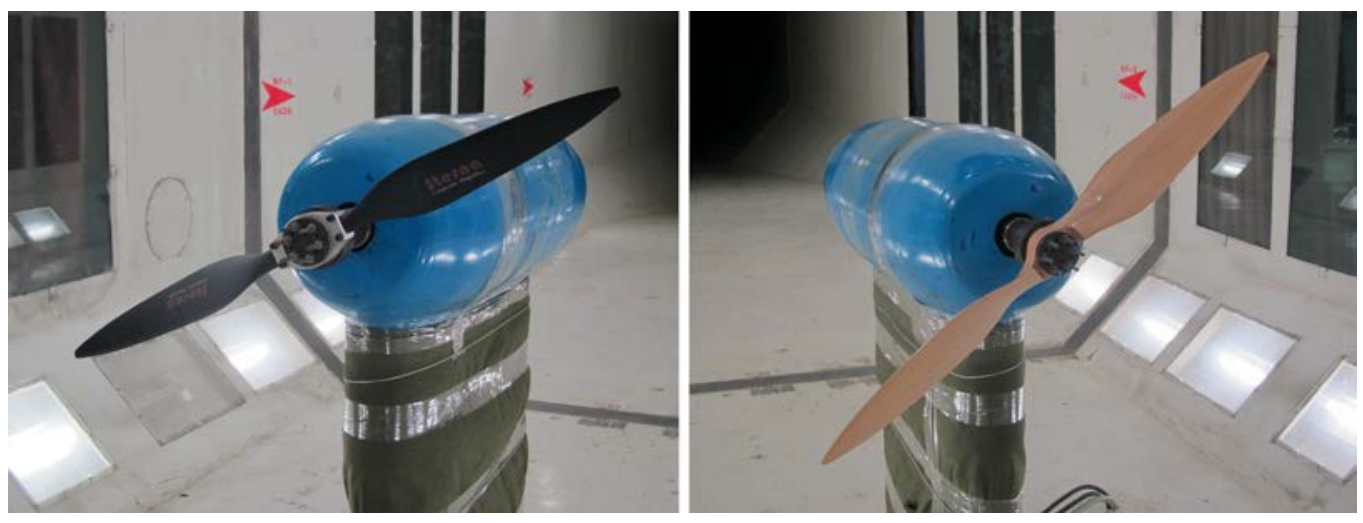

Fig. 1 1\#(left) carbon fiber and 2\#( right)woody propeller in wind tunnel

Propeller rotated with constant rotational speed $\mathrm{n}_{\mathrm{s}}=900 、 1200 、 1500 、 1800 、 2100 、 2400 、 2700$ 、 3000、3300、3600、3900 (rpm), wind velocity of the wind tunnel were $V=20 \mathrm{~m} / \mathrm{s}$ and $40 \mathrm{~m} / \mathrm{s}$.

Where $\rho$ is the density of the medium in which the propeller is operating.

$$
\rho=0.0034831 \times P /(273.15+t)
$$

The propeller advance ratio $\lambda$, a no dimensional term, is defined as

$$
\begin{aligned}
& \lambda=\frac{V}{n_{s} D} \\
& C_{T}=\frac{T}{\rho n_{s}^{2} D^{4}} \\
& C_{Q}=\frac{Q}{\rho n_{s}^{2} D^{5}} \\
& C_{W}=\frac{W}{\rho n_{s}^{3} D^{5}}
\end{aligned}
$$

Where $C_{T}$ is the thrust coefficient, $C_{Q}$ is the torque coefficient and $C_{W}$ is the coefficient of power of the propeller.

Propeller efficiency $\eta$ can be defined as the ratio of the power output to the power input:

$$
\eta=\frac{C_{T} \lambda}{C_{W}}
$$

\section{Data processing}

The propeller thrust $\mathrm{T}$, torque $\mathrm{Q}$, power $\mathrm{W}$ were obtained by experiments and the $C_{T}, C_{Q}$, and $C_{W}$ were calculated. The results are shown in Fig. 3-4 

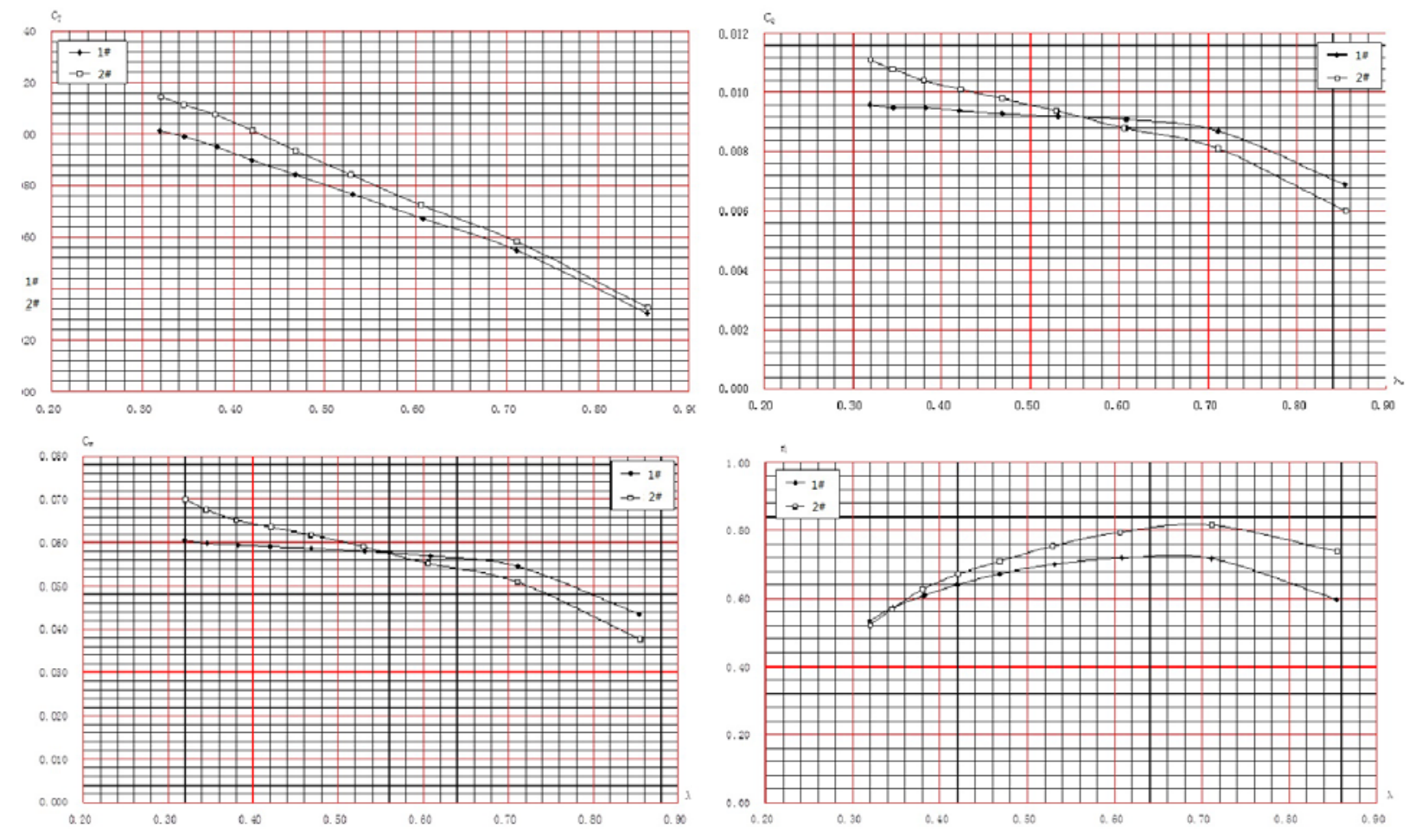

Fig. 3 The $C_{T}, C_{Q}, C_{W}$ and $\eta$ when velocity was $20 \mathrm{~m} / \mathrm{s}$
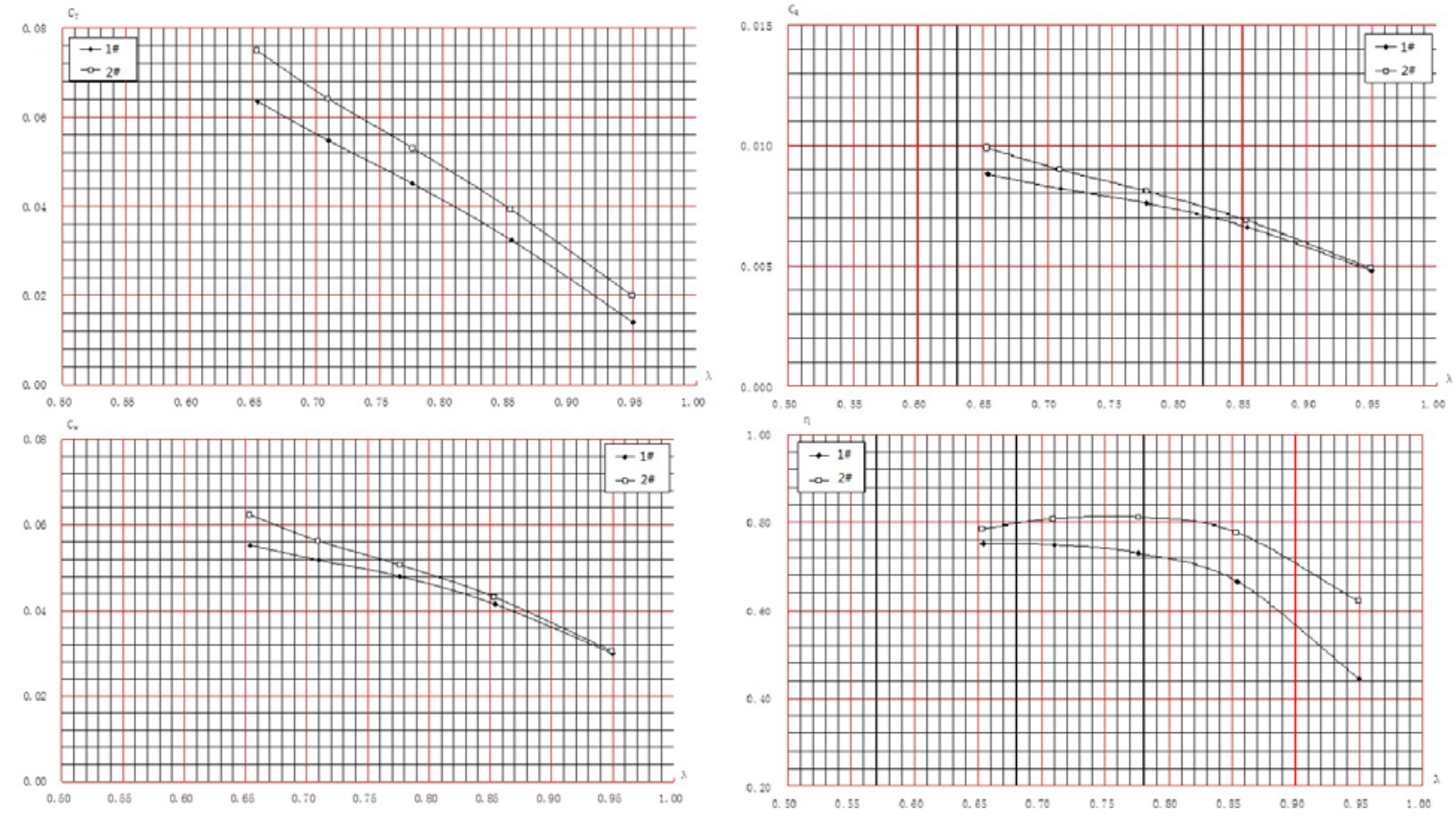

Fig. 4 The $C_{T}, C_{Q}, C_{W}$ and $\eta$ when velocity was $40 \mathrm{~m} / \mathrm{s}$

\section{Summary}

This paper analyses the influence of propeller root chord effect on aerodynamic characteristics. Through study based on the experimental results, we can obtained following conclusions:

- Due to the small radius, the velocity at the root is lower than the speed at the tip of propeller. This has the effect that the lift, torque located at the root of the blade is lower than other part of the blade. To maintain the lift near the blade root, it can increase the width of the propeller elements approaching the axis. 
- The efficiency $\eta$ of $1 \#$ propeller is $10 \%$ lower than $2 \#$ propeller. The Thrust $\mathrm{T}$ of 1 \# propeller is about $13 \%$ lower than 2\# propeller full range. Due to the effect of the root chord, the blade root may subject to the leakage of fluid due to the pressure on the lower surface and the depression on the upper surface. And the efficiency losses generated by the blade root leakage, may defeat the aerodynamic efficiency of the propeller.

- Combine the superiority in resistance of the carbon fiber material, and increasing the root chord within a reasonable range will increase the performance of propeller.

\section{Acknowledgments}

This project was supported partially by Science and Technology Department Foundation project of Liaoning Province (No. 2015020167) and Liaoning BaiQianWan Talents Program (No. 2014921048)

\section{References}

[1] H. Ghassemi, A. Mardan, Numerical Analysis of Hub Effect on Hydrodynamic Performance of Propellers with Inclusion of PBCF to Equalize the Induced Velocity, J. POLISH MARITIME RESEARCH 2(73) 2012 Vol. 19; pp. 17-24

[2] Larrabee E. Practical design of minimum Induced Loss Propellers [C]. SAE Preprint 790585, 1979.

[3] Eppler R, Hepperle M. A procedure for propeller design by inverse methods [C]. Proceedings of the International Conference on Inverse Design Concepts in Engineering Sciences (ICIDES), pp. 445-460, Austin TX, October 17-18, 1984.

[4] Angelo S D, Berardi F, Menisci E. Aerodynamic performances of propellers with parametric considerations on the optimal design [J]. The Aeronautical journal, 2002, 106(1060):313-320

[5] XIANG Song, ZHANG Li-guo, TONG Sheng-xi, Wu Jiang, LIU Yuan-qiang. A design method for high efficiency propeller [J]. Journal of Aerospace Power, 2015, 30(1):136-141.

[6] LIU Yuan-qiang, GUO Jin-suo, XIANG Song, TONG Sheng-xi, JIANG Wen-hui. Aerodynamic performances calculation of propellers based on standard strip analysis [J]. Journal of Shenyang Aerospace University, 2013, 30(1):43-46.

[7] A. B. HAINES, B.Sc., AND P. B. CHATER, 24-ft. Tunnel Tests on a rotor Wooden Spitfire Propeller. Test Results, and Data for Single Radius Calculations [J] A.R.C. Technical Report, R. \& M. No. 2357

[8] J. Cecrdle, Influence of Propeller Blade Lift Distribution on Whirl Flutter Stability Characteristics[C]World Academy of Science, Engineering and Technology International Journal of Mechanical, Aerospace, Industrial, Mechatronic and Manufacturing Engineering Vol:8, No:4, 2014 